\title{
Localization and Time Synchronization in Wireless Sensor Networks: A Unified Approach
}

\author{
Jun Zheng and Yik-Chung $\mathrm{Wu}$ \\ Department of Electrical and Electronic Engineering \\ The University of Hong Kong, Hong Kong \\ Email: \{zhengjun, ycwu\}@eee.hku.hk
}

\begin{abstract}
Localization and synchronization are two important issues in communication systems and wireless sensor networks. These two problems are addressed in many applications, and share many aspects in common. However, these two problems are traditionally treated separately. In this paper, we present a unified framework to jointly solve these two problems at the same time. The maximum likelihood joint estimation of location and timing is firstly derived. Then, a more computationally efficient twostage least square method is proposed. The Cramer-Rao lower bound for the joint localization and time synchronization is also derived. Simulation results show that the performances of the maximum likelihood and two-stage least square estimators can both achieve the Cramer-Rao lower bound.
\end{abstract}

\section{INTRODUCTION}

Localization is an important application in wireless communications, sensor networks, radar and sonar [1], [2]. It has been intensively studied by the signal processing community in the past few decades, especially after the Federal Communications Commission (FCC) issued the order of emergency 911 (E-911) [3]. Conventional source localization are based on time of arrival (TOA), time difference of arrival (TDOA), angle of arrival (AOA) or received signal strength (RSS) [2]. The TOA and TDOA based approaches strongly depends on the assumption that time synchronization has already been achieved.

On the other hand, time synchronization are traditionally studied by the computer science community from protocol design point of view. Many time synchronization protocols have been proposed for computer networks and wireless sensor networks, such as the Network Time Protocol (NTP), Timingsync Protocol for Sensor Networks (TPSN) and Reference Broadcast Synchronization (RBS) [4]. Recently, Noh et al mathematically evaluated the performance of two-way message exchange time synchronization method in wireless sensor networks [5].

In this paper, we propose to jointly solve the problems of localization and time synchronization in wireless sensor networks from the signal processing perspective. Some pioneer researchers have noticed the similarities between the problem of source localization and time synchronization [6], [7]. However, they only pointed out the links between the two problems and explored the possibility of jointly implementing localization and synchronization in the protocol level. To the best of our knowledge, this is the first work that mathematically models and analyzes the joint localization and synchronization problem from signal processing point of view.
The rest of this paper is organized as follows. The system model is introduced in Section II. In Section III, the maximum likelihood estimator for joint location and time parameters estimation is derived. A two-stage least square approach is then proposed in Section IV. The Cramer-Rao lower bound (CRLB) for the joint estimation problem is derived in Section V. Computer simulations are presented in Section VI, and conclusions are drawn in Section VII.

\section{SySTEM MODEL}

We consider single node joint localization and synchronization in a wireless sensor network, where only one node needs to be localized and synchronized to the anchors at a time. In this system, we assume that there are $L(L \geq 3)$ anchors $A_{l}$ with known location at $\left(a_{x l}, a_{y l}\right) l=1,2, \cdots, L$ and the anchors are synchronized to each other. A node $P$ exchanges messages with anchors and use the information in the exchanged messages to estimate its location and synchronizes itself to the anchors.

The information communicated between the node and the anchors is the time-stamps in two-way message exchanges [5]. Assuming there are $M$ message exchanges in total, the $m$ th message is sent at time $T_{l m}$ and it is received at time $R_{l m}$, as shown in Figure 1. For simplicity, we assume the packet is sent from the anchor to the node when $m$ is odd, and from the node to the anchor when $m$ is even. Note that $T_{l(2 k+1)}$ and $R_{l(2 k)}$ are measured with respect to the clock of anchors, while $T_{l(2 k)}$ and $R_{l(2 k+1)}$ are measured with respect to the clock of node $P$, where $k$ is an integer. Considering both the clock offset $\theta_{0}$ and the clock skew $\theta_{s}$ between node $P$ and the anchors, the exchanged time-stamps can be modeled as [8]

$$
\begin{gathered}
R_{l m}=\theta_{s}\left(T_{l m}+t_{l}+n_{l m}\right)+\theta_{0} \\
\quad l=1,2, \cdots, L, m=1,3, \cdots, M-1 \\
T_{l m}=\theta_{s}\left(R_{l m}-t_{l}-n_{l m}\right)+\theta_{0} \\
\quad l=1,2, \cdots, L, m=2,4, \cdots, M
\end{gathered}
$$

where $t_{l}=\frac{1}{c}\left\|\mathbf{x}-\mathbf{a}_{l}\right\|$ is the propagation delay between node $P$ and anchor $A_{l}$, with $c$ the speed of light. The location vector of node $P$ is defined as $\mathbf{x}=[x, y]^{T}$, and that for anchor $A_{l}$ is $\mathbf{a}_{l}=\left[a_{x l}, a_{y l}\right]^{T} . n_{l m}$ is the measurement uncertainties due to the sending, receiving and accessing delay at the sending and receiving end [9]. In general, this delay can be modeled as random variables from Gaussian, exponential, 
Gamma, or Weibull distribution depending on the scenario [5]. In this paper, we focus on the case when $n_{l m}$ is modeled as independent Gaussian random variables with zero mean and variance of $\sigma^{2}$.

\section{Maximum Likelihood Joint Estimation}

We divide both sides of (1) and (2) by $\theta_{s}$ and introduce two new variables $\theta_{1} \triangleq \frac{1}{\theta_{s}}$ and $\theta_{2} \triangleq \frac{\theta_{0}}{\theta_{s}}$, then we have

$$
\begin{gathered}
T_{l m}+t_{l}=R_{l m} \theta_{1}-\theta_{2}-n_{l m} \\
\quad l=1,2, \cdots, L, m=1,3, \cdots, M-1 \\
R_{l m}-t_{l}=T_{l m} \theta_{1}-\theta_{2}+n_{l m} \\
\quad l=1,2, \cdots, L, m=2,4, \cdots, M .
\end{gathered}
$$

Introducing $\boldsymbol{\theta}=\left[\begin{array}{ll}\theta_{1} & \theta_{2}\end{array}\right]^{T}$, we can put the equations in (3) and (4) into a matrix form

$$
\mathbf{T}(\mathbf{x})=\mathbf{R} \boldsymbol{\theta}+\mathbf{n}
$$

where

$$
\mathbf{T}(\mathbf{x})=\left[\begin{array}{c}
T_{11}+t_{1} \\
R_{12}-t_{1} \\
\vdots \\
T_{21}+t_{2} \\
R_{21}-t_{2} \\
\vdots
\end{array}\right], \mathbf{R}=\left[\begin{array}{cc}
R_{11} & -1 \\
T_{12} & -1 \\
\vdots & \vdots \\
R_{21} & -1 \\
T_{21} & -1 \\
\vdots & \vdots
\end{array}\right] \text { and } \mathbf{n}=\left[\begin{array}{c}
-n_{11} \\
n_{12} \\
\vdots \\
-n_{21} \\
n_{22} \\
\vdots
\end{array}\right]
$$

Given the Gaussian noise, the maximum likelihood (ML) estimate of $\mathrm{x}$ and $\boldsymbol{\theta}$ can be obtained by minimizing [10]

$$
\Lambda(\tilde{\mathbf{x}}, \tilde{\boldsymbol{\theta}})=(\mathbf{T}(\tilde{\mathbf{x}})-\mathbf{R} \tilde{\boldsymbol{\theta}})^{T}(\mathbf{T}(\tilde{\mathbf{x}})-\mathbf{R} \tilde{\boldsymbol{\theta}}),
$$

where $\tilde{\mathbf{x}}$ and $\tilde{\boldsymbol{\theta}}$ are the trial values for $\mathbf{x}$ and $\boldsymbol{\theta}$. When $\tilde{\mathbf{x}}$ is fixed, solving $\boldsymbol{\theta}$ from (5), we have

$$
\hat{\boldsymbol{\theta}}=\left(\mathbf{R}^{T} \mathbf{C}^{-1} \mathbf{R}\right)^{-1} \mathbf{R}^{T} \mathbf{C}^{-1} \mathbf{T}(\tilde{\mathbf{x}})
$$

where $\mathbf{C}=\sigma^{2} \mathbf{I}$ is the covariance matrix of the noise vector $\mathbf{n}$. Inserting (7) back to (6), after some straightforward manipulation, the location $\mathrm{x}$ can be estimated as

$$
\hat{\mathbf{x}}=\arg \min _{\mathbf{x}}\left\{\|\mathbf{T}(\mathbf{x})\|^{2}-\left\|\mathbf{P}^{\prime} \mathbf{T}(\mathbf{x})\right\|^{2}\right\}
$$

where $\mathbf{P}^{\prime}=\mathbf{R}\left(\mathbf{R}^{T} \mathbf{R}\right)^{-1} \mathbf{R}^{T}$ is the projection matrix.

Let $\mathbf{P}=\mathbf{I}-\mathbf{P}^{\prime}$, (8) becomes

$$
\hat{\mathbf{x}}=\arg \min _{\mathbf{x}}\left\{=\|\mathbf{P T}(\mathbf{x})\|^{2}\right\} .
$$

The final estimate of $\boldsymbol{\theta}$ is obtained by inserting the result of (9) back to (7). The two dimensional optimization problem in (9) can be solved by alternating projection as has been reported in [11].

\section{Two-STage Least Square Approach}

The above ML method involves solving a two-dimensional nonlinear optimization problem, which is computationally expensive. In this section, we propose a two-step least square (LS) approach, in which closed form solution exists, to estimate the location and timing parameters.

\section{A. Step 1: Least Square Approximation}

First, from (3) and (4), we have

$$
\begin{gathered}
t_{l}=\frac{1}{c} \sqrt{\left(x-a_{x l}\right)^{2}+\left(y-a_{y l}\right)^{2}}=R_{l m} \theta_{1}-\theta_{2}-T_{l m}-n_{l m} \\
\quad l=1,2, \cdots, L, m=1,3, \cdots, M-1 \\
t_{l}=\frac{1}{c} \sqrt{\left(x-a_{x l}\right)^{2}+\left(y-a_{y l}\right)^{2}}=-T_{l m} \theta_{1}+\theta_{2}+R_{l m}-n_{l m} \\
\quad l=1,2, \cdots, L, m=2,4, \cdots, M .
\end{gathered}
$$

Squaring the above two equations and re-arranging them, we have

$$
\begin{gathered}
\frac{2}{c^{2}}\left(a_{x l} x+a_{y l} y\right)-2 T_{l m} R_{l m} \theta_{1}+2 T_{l m} \theta_{2}+R_{l m}^{2} \theta_{1}^{2}-2 R_{l m} \theta_{1} \theta_{2} \\
+\left(\theta_{2}^{2}-\frac{1}{c^{2}}\left(x^{2}+y^{2}\right)\right)=\frac{1}{c^{2}}\left(a_{x l}^{2}+a_{y l}^{2}\right)-T_{l m}^{2}+e_{l m}, \\
l=1,2, \cdots, L, m=1,3, \cdots, M-1 \\
\frac{2}{c^{2}}\left(a_{x l} x+a_{y l} y\right)-2 T_{l m} R_{l m} \theta_{1}+2 R_{l m} \theta_{2}+T_{l m}^{2} \theta_{1}^{2}-2 T_{l m} \theta_{1} \theta_{2} \\
+\left(\theta_{2}^{2}-\frac{1}{c^{2}}\left(x^{2}+y^{2}\right)\right)=\frac{1}{c^{2}}\left(a_{x l}^{2}+a_{y l}^{2}\right)-R_{l m}^{2}+e_{l m}, \\
l=1,2, \cdots, L, m=2,4, \cdots, M
\end{gathered}
$$

where

$$
e_{l m}= \begin{cases}2\left(R_{l m} \theta_{1}-\theta_{2}-T_{l m}\right) n_{l m}-n_{l m}^{2}, & m \text { odd } \\ 2\left(-T_{l m} \theta_{1}+\theta_{2}+R_{l m}\right) n_{l m}-n_{l m}^{2}, & m \text { even }\end{cases}
$$

includes all the terms involving $n_{l m}$, which can also be interpreted as the equation error.

By introducing three additional variables

$$
\xi_{5}=\theta_{1}^{2}, \xi_{6}=\theta_{2}^{2}-\frac{1}{c^{2}}\left(x^{2}+y^{2}\right) \text { and } \xi_{7}=\theta_{1} \theta_{2},
$$

and defining a new variable vector $\boldsymbol{\xi}=\left[\mathbf{x}^{T}, \boldsymbol{\theta}^{T}, \xi_{5}, \xi_{6}, \xi_{7}\right]^{T}$, we can put (12) and (13) into a matrix form as

$$
B \xi=\mathrm{b}+\mathrm{e}
$$

where

$$
\begin{gathered}
\boldsymbol{B}=2\left[\begin{array}{ccccccc}
\frac{a_{x 1}}{c^{2}} & \frac{a_{y 1}}{c^{2}} & -T_{11} R_{11} & T_{11} & \frac{1}{2} R_{11}^{2} & \frac{1}{2} & -R_{11} \\
\frac{a_{x 1}}{c^{2}} & \frac{a_{y 1}}{c^{2}} & -T_{12} R_{12} & R_{12} & \frac{1}{2} T_{12}^{2} & \frac{1}{2} & -T_{12} \\
& & \vdots & & & \\
\frac{a_{x 2}}{c^{2}} & \frac{a_{y 2}}{c^{2}} & -T_{21} R_{21} & T_{21} & \frac{1}{2} R_{21}^{2} & \frac{1}{2} & -R_{21} \\
\frac{a_{x 2}}{c^{2}} & \frac{a_{y 2}}{c^{2}} & -T_{22} R_{22} & R_{22} & \frac{1}{2} T_{22}^{2} & \frac{1}{2} & -T_{22} \\
& \vdots & & &
\end{array}\right] \\
\boldsymbol{b}=\left[\begin{array}{c}
\left(a_{x 1}^{2}+a_{y 1}^{2}\right) / c^{2}-T_{11}^{2} \\
\left(a_{x 1}^{2}+a_{y 1}^{2}\right) / c^{2}-R_{12}^{2} \\
\vdots \\
\left(a_{x 2}^{2}+a_{y 2}^{2}\right) / c^{2}-T_{21}^{2} \\
\left(a_{x 2}^{2}+a_{y 2}^{2}\right) / c^{2}-R_{22}^{2} \\
\vdots
\end{array}\right]
\end{gathered}
$$

and $\mathbf{e}$ is the noise vector formed from $e_{l m}$.

Solving (16), we can obtain the estimates of $\boldsymbol{\xi}$ by

$$
\hat{\boldsymbol{\xi}}_{L S}=\left(\mathbf{B}^{T} \mathbf{B}\right)^{-1} \mathbf{B}^{T} \mathbf{b} .
$$


However, (17) only provides an rough estimate of the desired variables $\boldsymbol{x}$ and $\boldsymbol{\theta}$, because of two reasons. First, the estimator (17) assumes all the items in the measurement vector b have same variances, while it is obviously not the case as can be seen from (14). Secondly, it does not include the constraints between the elements of $\boldsymbol{\xi}$, and thus the estimate may be inconsistent. Next, we propose a second step to improve the estimate of (17) based on the above two observations.

\section{B. Step 2: Constrained Weighted Least Square}

The covariance matrix of the noise vector $\mathbf{e}$ can be derived from (14) as $\mathbf{Q}_{\mathbf{e}}=4 \sigma^{2} \operatorname{diag}\left(\left(R_{11} \theta_{1}-\theta_{2}-T_{11}\right)^{2},\left(\theta_{2}-T_{12} \theta_{1}+\right.\right.$ $\left.\left.R_{12}\right)^{2} \cdots\right)$, where we ignored the second order term of noise because $n_{l m}$ is usually very small. Notice that $\mathbf{Q}_{\mathbf{e}}$ depends on $\theta_{1}$ and $\theta_{2}$, which are also parameters of interest. Fortunately, we can use the rough estimate in (17) of step 1. The weighted Least Square solution of (16) is then given by

$$
\hat{\boldsymbol{\xi}}_{W L S}=\left(\mathbf{B}^{T} \hat{\mathbf{Q}}_{\mathbf{e}}^{-1} \mathbf{B}\right)^{-1} \mathbf{B}^{T} \hat{\mathbf{Q}}_{\mathbf{e}}^{-1} \mathbf{b},
$$

where $\hat{\mathbf{Q}}_{\mathbf{e}}$ is $\mathbf{Q}_{\mathbf{e}}$ with $\theta_{1}$ and $\theta_{2}$ replaced by $\hat{\theta}_{1}$ and $\hat{\theta}_{2}$ from (17), respectively.

Next, we employ the relationships between elements of $\boldsymbol{\xi}$ given by (15), which can be put into the following matrix form

$$
\mathbf{G} \boldsymbol{\omega}=\hat{\boldsymbol{\xi}}_{W L S}+\boldsymbol{\eta}
$$

where

$$
\boldsymbol{\omega}=\left[\begin{array}{l}
\mathbf{x} \\
\boldsymbol{\theta}
\end{array}\right], \quad \mathbf{G}=\left[\begin{array}{l}
\mathbf{I}_{4} \\
\tilde{\mathbf{G}}
\end{array}\right], \quad \tilde{\mathbf{G}}=\left[\begin{array}{cccc}
0 & 0 & \hat{\theta}_{1} & 0 \\
-\frac{\hat{x}}{c^{2}} & -\frac{\hat{y}}{c^{2}} & 0 & \hat{\theta}_{2} \\
0 & 0 & \frac{\hat{\theta}_{2}}{2} & \frac{\hat{\theta}_{1}}{2}
\end{array}\right]
$$

and $\boldsymbol{\eta}$ is the estimation error in (18). Together with the fact that the estimation covariance of (18) is given by [10] $\mathrm{Q}_{\xi}=$ $\left(\mathbf{B}^{T} \hat{\mathbf{Q}}_{\mathbf{e}}^{-1} \mathbf{B}\right)^{-1}$, the weighted least square solution of (19) is

$$
\hat{\boldsymbol{\omega}}=\left(\mathbf{G}^{T} \mathbf{Q}_{\boldsymbol{\xi}}^{-1} \mathbf{G}\right)^{-1} \mathbf{G}^{T} \mathbf{Q}_{\boldsymbol{\xi}}^{-1} \hat{\boldsymbol{\xi}}_{W L S} .
$$

Notice that the proposed CWLS estimator (21) is in close form and does not need a searching step as in the ML estimator (9).

\section{CRAmer-Rao Lower Bound}

In order to provide a performance reference for the proposed estimators, we derive the CRLB for the joint estimation problem in this section.

From (1) and (2) we have

$$
\begin{gathered}
T_{l m}=\frac{1}{\theta_{s}}\left(R_{l m}-\theta_{0}\right)-\frac{1}{c} \sqrt{\left(x-a_{x l}\right)^{2}+\left(y-a_{y l}\right)^{2}}-n_{l m} \\
\quad l=1,2, \cdots, L, m=1,3, \cdots, M-1 \\
R_{l m}=\frac{1}{\theta_{s}}\left(T_{l m}-\theta_{0}\right)+\frac{1}{c} \sqrt{\left(x-a_{x l}\right)^{2}+\left(y-a_{y l}\right)^{2}}+n_{l m} \\
\quad l=1,2, \cdots, L, m=2,4, \cdots, M .
\end{gathered}
$$

Notice that $\mathbf{Z}=\left[T_{11}, R_{12}, \cdots, T_{21}, R_{22}, \cdots\right]^{T}$ is a Gaussian vector with mean $\boldsymbol{\mu}(\boldsymbol{\Phi})$ and covariance matrix $C$, where we have defined $\mathbf{\Phi}=\left[x, y, \theta_{s}, \theta_{0}\right]^{T}$, and the mean vector is

$$
\boldsymbol{\mu}(\boldsymbol{\Phi})=\left[\begin{array}{c}
\left(R_{11}-\theta_{0}\right) / \theta_{s}-t_{1} \\
\left(T_{12}-\theta_{0}\right) / \theta_{s}+t_{1} \\
\vdots \\
\left(R_{21}-\theta_{0}\right) / \theta_{s}-t_{2} \\
\left(T_{22}-\theta_{0}\right) / \theta_{s}+t_{2} \\
\vdots
\end{array}\right] .
$$

The variance of the estimation of $\Phi$ is bound by the CRLB, which is given by [10]

$$
\operatorname{CRLB}(\boldsymbol{\Phi})=\left(\frac{\partial \boldsymbol{\mu}^{T}}{\partial \boldsymbol{\Phi}} \mathbf{C}^{-1} \frac{\partial \boldsymbol{\mu}}{\partial \boldsymbol{\Phi}^{T}}\right)^{-1} .
$$

The partial derivative can be computed as

$$
\frac{\partial \boldsymbol{\mu}}{\partial \boldsymbol{\Phi}^{T}}=\left[\begin{array}{cccc}
-\frac{x-a_{x 1}}{c d_{1}} & -\frac{y-a_{y 1}}{c d_{1}} & -\frac{R_{11}-\theta_{0}}{\theta_{s}^{2}} & -\frac{1}{\theta_{s}} \\
\frac{x-a_{x 1}}{c d_{1}} & \frac{y-a_{y 1}}{c d_{1}} & -\frac{T_{12}-\theta_{0}}{\theta_{s}^{2}} & -\frac{1}{\theta_{s}} \\
& \vdots & \vdots & \\
-\frac{x-a_{x 2}}{c d_{2}} & -\frac{y-a_{y 2}}{c d_{2}} & -\frac{R_{21}-\theta_{0}}{\theta_{s}^{2}} & -\frac{1}{\theta_{s}} \\
\frac{x-a_{x 2}}{c d_{2}} & \frac{y-a_{y 2}}{c d_{2}} & -\frac{T_{22}-\theta_{0}}{\theta_{s}^{2}} & -\frac{1}{\theta_{s}} \\
& \vdots & \vdots &
\end{array}\right] \triangleq \mathbf{A} .
$$

With $\mathbf{C}=\sigma^{2} \mathbf{I}$, we have

$$
\operatorname{CRLB}(\boldsymbol{\Phi})=\sigma^{2}\left(\mathbf{A}^{T} \mathbf{A}\right)^{-1} .
$$

The CRLB of the $k$ th parameter in $\boldsymbol{\Phi}$ is then given by the $(k, k)$ th element of $\sigma^{2}\left(\mathbf{A}^{T} \mathbf{A}\right)^{-1}$.

Remark: The CRLB of location estimate with perfect timing is given by $\sigma^{2}\left(\mathbf{A}_{12}^{T} \mathbf{A}_{12}\right)^{-1}$, and the CRLB of timing estimate with perfect location is given by $\sigma^{2}\left(\mathbf{A}_{34}^{T} \mathbf{A}_{34}\right)^{-1}$, where $\mathbf{A}_{12}$ and $\mathbf{A}_{34}$ are the first two columns and the last two columns of $\mathbf{A}$, respectively.

\section{Simulation Results and Discussions}

In this section, simulation results are presented to verify the effectiveness of the proposed schemes. In the computer simulations, there are four anchors located at $(-50,30)$, $(-40,-90),(90,-60)$ and $(20,70)$. The coordinates of node $P$ is randomly drawn from $[-30,30]$. The clock offset and clock skew are normally distributed with mean 0 and 1 , respectively, according to [9]. The mean square error (MSE) of the location is defined as $E\left\{(x-\hat{x})^{2}+(y-\hat{y})^{2}\right\}$, where $E\{\cdot\}$ is the expectation with respect to the realizations of $\hat{x}$ and $\hat{y}$. All simulations are average of 1000 independent runs.

We set the number of message exchanges $(M)$ between the anchors and node $P$ to 6 , and examine the effect of signal-tonoise ratio (SNR, defined as $1 / \sigma^{2}$ ) on the estimation performance. Figure 2 shows the MSE of the location estimate of ML in (9), LS in (17), CWLS in (21), and localization without considering timing synchronization. They are compared with the CRLB for the joint localization and synchronization, and that for localization when we have perfect timing. It can be seen that the proposed ML and CWLS methods can both attain 
the CRLB and the localization fails if we don't consider the timing synchronization. Furthermore, the CRLB of the joint localization and synchronization is very close to the CRLB when we have perfect timing, which implies that we have little performance loss when estimating the location and timing jointly. This conclusion also applies to the clock offset and clock skew estimation as can be seen from Figures 3 and 4 .

\section{CONCLUSions ANd Future Work}

In this paper, we investigated the similarities between localization and time synchronization in wireless sensor networks. We proposed a maximum likelihood (ML) joint localization and synchronization scheme, and also a two-stage least square (LS) estimator. Computer simulation showed that the proposed ML and two-stage LS methods can both attain the CramerRao lower bound. Our ongoing research includes extending the above joint localization and synchronization concept to network-wide localization and synchronization, and investigating the effect of non-line of sight on the joint estimation.

\section{REFERENCES}

[1] J. Caffery and G. Stuber, "Subscriber locaiton in CDMA cellular Networks," IEEE Tran. on Vehicular Tech. vol.47, no.2, May 1998.

[2] G. Mao, B. Fidan, and B. Anderson, "Wireless sensor network localization techniques," Computer Networks, vol.51, no.10, Jul. 2007.

[3] Y. Zhao, "Standardization of mobile phone position for 3G systems," IEEE Commun. Mag. vol.40, no.7, pp.108-116, Jul. 2002.

[4] B. Sundararaman, U. Buy, and A. Kshemkalyani, "Clock synchronization for wireless sensor networks: a survey," Ad Hoc Networks, vol,3, no.3, pp.281-323, May 2005.

[5] K. Noh, Q. M. Chaudhari, E. Serpedin and B. W. Suter, "Novel clock phase offset and skew estimation using two-way timing message exchanges for wireless sensor networks," IEEE Trans. on Communications, vol.55, no.4, pp.766-777, 2007.

[6] K. Romer and F. Mattern, "Towards a unified view on space and time in sensor networks," Computer Communications, vol.28, Aug. 2005

[7] H. Oliveira, E. Nakamura, A. Loureiro, "Localization in time and space for sensor networks." Proc. 21st International Conf. on Advanced Info. Net. and App., pp.539-546, May 2007.

[8] N.Freris, P. Kumar, "Fundamental limits on synchronization of affine clocks in networks," 46th IEEE Conf. on Decision and Ctrl, Dec. 2007

[9] J. Elson, L. Girod, and D. Estrin, "Fine-grained network time synchronization using reference broadcasts," in Proc. 5th Operating Syst. Design and Implementation Symp., pp.147 - 163, Dec. 2002.

[10] S. Kay, Fundamentals of Statistical Signal Processing: Estimation Theory, Englewood Cliffs, NJ: Prentice-Hall, 1993.

[11] I. Ziskind and M. Wax, "Maximum likelihood localization of multiple sources by alternating projection," IEEE Trans. on Signal Processing, vol.36, no.10, pp.1553-1560, 1998.

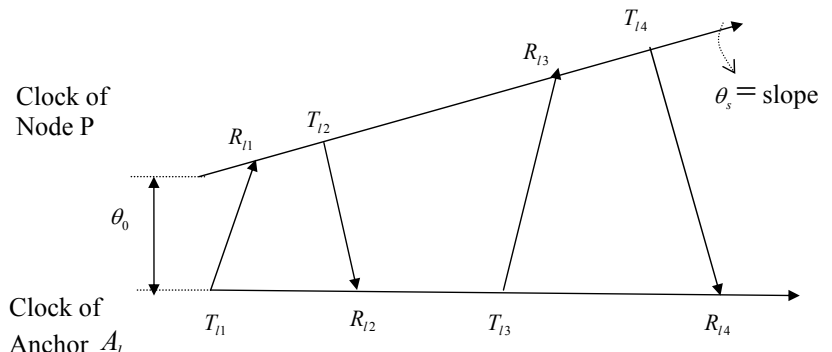

Fig. 1. Two-way message exchange between node $\mathrm{P}$ and the anchor

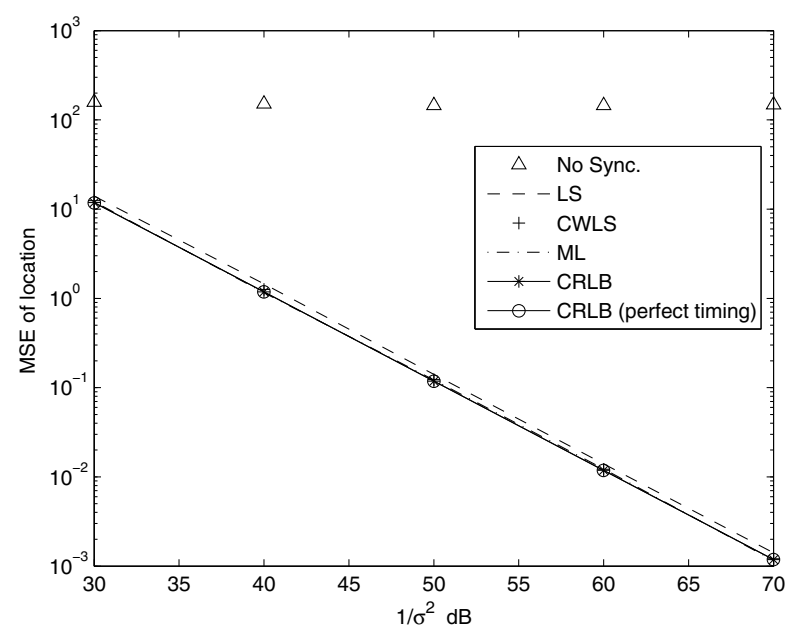

Fig. 2. MSE of location versus SNR

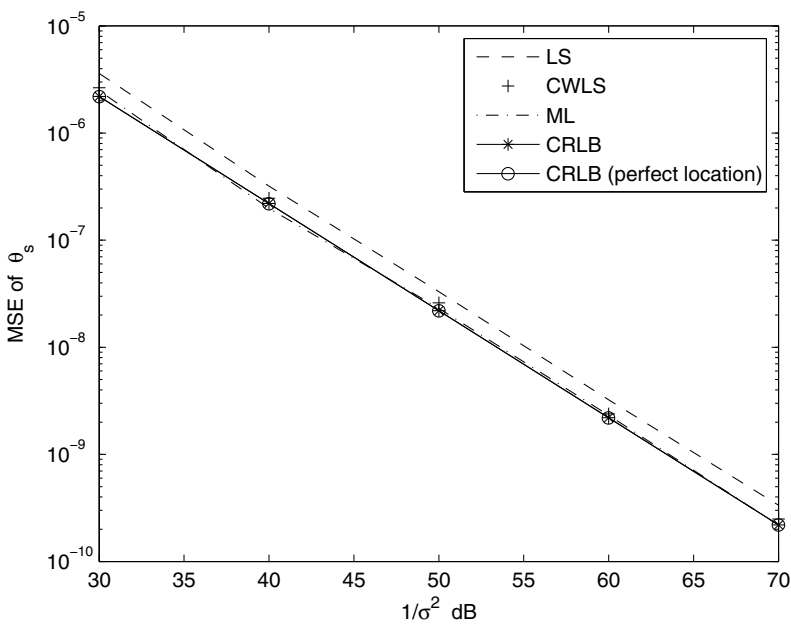

Fig. 3. MSE of $\theta_{s}$ versus SNR

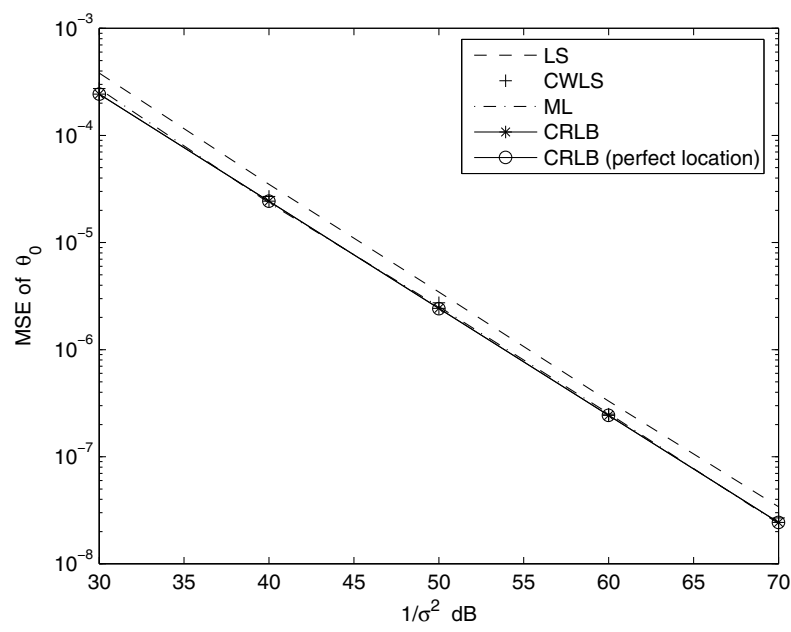

Fig. 4. MSE of $\theta_{0}$ versus SNR 